\title{
High surface magnetic field in red giants as a new signature of planet engulfment?
}

\author{
Giovanni Privitera ${ }^{1,2}$, Georges Meynet ${ }^{1}$, Patrick Eggenberger ${ }^{1}$, Cyril Georgy $^{1}$, Sylvia Ekström ${ }^{1}$, \\ Aline A. Vidotto ${ }^{3}$, Michele Bianda $^{2}$, Eva Villaver ${ }^{4}$, and Asif ud-Doula ${ }^{5}$ \\ ${ }^{1}$ Geneva Observatory, University of Geneva, Ch. Maillettes 51, 1290 Sauverny, Switzerland \\ e-mail: giovanni.privitera@unige.ch \\ 2 Istituto Ricerche Solari Locarno, Via Patocchi, 6605 Locarno-Monti, Switzerland \\ 3 School of Physics, Trinity College Dublin, The University of Dublin, Ireland \\ 4 Department of Theoretical Physics, Universidad Autónoma de Madrid, Módulo 8, 28049 Madrid, Spain \\ 5 Penn State Worthington Scranton, Dunmore, PA 18512, USA
}

Received 17 June 2016 / Accepted 31 August 2016

\begin{abstract}
Context. Red giant stars may engulf planets. This may increase the rotation rate of their convective envelope, which could lead to strong dynamo-triggered magnetic fields.

Aims. We explore the possibility of generating magnetic fields in red giants that have gone through the process of a planet engulfment. We compare them with similar models that evolve without any planets. We discuss the impact of magnetic braking through stellar wind on the evolution of the surface velocity of the parent star.

Methods. By studying rotating stellar models with and without planets and an empirical relation between the Rossby number and the surface magnetic field, we deduced the evolution of the surface magnetic field along the red giant branch. The effects of stellar wind magnetic braking were explored using a relation deduced from magnetohydrodynamics simulations.

Results. The stellar evolution model of a red giant with $1.7 M_{\odot}$ without planet engulfment and with a time-averaged rotation velocity during the main sequence equal to $100 \mathrm{~km} \mathrm{~s}^{-1}$ shows a surface magnetic field triggered by convection that is stronger than $10 \mathrm{G}$ only at the base of the red giant branch, that is, for gravities $\log g>3$. When a planet engulfment occurs, this magnetic field can also appear at much lower gravities, that is, at much higher luminosities along the red giant branch. The engulfment of a $15 M_{\mathrm{J}}$ planet typically produces a dynamo-triggered magnetic field stronger than $10 \mathrm{G}$ for gravities between 2.5 and 1.9 . We show that for reasonable magnetic braking laws for the wind, the high surface velocity reached after a planet engulfment may be maintained sufficiently long to be observable.

Conclusions. High surface magnetic fields for red giants in the upper part of the red giant branch are a strong indication of a planet engulfment or of an interaction with a companion. Our theory can be tested by observing fast-rotating red giants such as HD 31994 , Tyc 0347-00762-1, Tyc 5904-00513-1, and Tyc 6054-01204-1 and by determining whether they show magnetic fields.
\end{abstract}

Key words. planet-star interactions - stars: magnetic field - stars: rotation

\section{Introduction}

The tidal forces between star and planets change the planetary orbit and may sometimes produce the engulfment of the planet by the star. A number of past studies (Soker et al. 1984; Siess \& Livio 1999a,b; Villaver \& Livio 2007, 2009; Nordhaus et al. 2010; Kunitomo et al. 2011; Villaver et al. 2014) have shown that the possible impact of an engulfment, planetary or otherwise, leads to changes in the stellar luminosity and radii for a short period of evolutionary time, to modifications of the surface abundances, in particular to an increase in lithium abundance (Alexander 1967; Fekel \& Balachandran 1993; Sandquist et al. 1998, 2002; Carlberg et al. 2010; Adamów et al. 2012), and to changes in the surface velocity (Carlberg et al. 2009; Carlberg 2014).

Our team focused on this last consequence in two previous papers referred to here as Papers I and II (Privitera et al. 2016a,b). To explore this effect, we used stellar rotating models where the rotation in the whole star can be followed consistently according to the shellular theory by Zahn (1992) and affected by tidal interaction when an external convective zone appears (Zahn 1977, 1989). Papers I and II showed that the observed surface velocities of some stars can only be explained by some interaction with a planet or a brown dwarf.

In this letter we study to which extent such tidal interactions and engulfment processes may trigger a magnetic field and also whether this planet-induced magnetic field might be strong enough to slow down the stellar rotation through the process of wind magnetic braking (ud-Doula \& Owocki 2002; ud-Doula et al. 2008). Section 2 briefly recalls the main ingredients of the models. The evolution of the Rossby number for different stellar models is discussed in Sect. 3. Planet-induced magnetic fields and their possible consequences on the evolution of the stellar rotation are presented in Sect. 4, and conclusions are drawn in Sect. 5.

\section{Physics of the models}

To study the problem addressed here, we need to understand stellar and planetary models whose evolutions are closely 
inter-linked through five different processes: (a) the evolution of the planetary orbit that accounts for the evolution of the star, (b) the changes in angular momentum of the star that are due to changes in planetary orbit, (c) the physics of the engulfment, (d) the link between the engulfment and the generation of a surface magnetic field, and (e) the impact of the generated magnetic field on the rotational evolution of the red giant star. The first three processes were extensively discussed in Papers I and II. Here we focus on the last two, which involve magnetic fields. Red giants are characterized by a deep convective envelope in slow rotation, therefore tidal dissipation can be introduced by taking into account only the equilibrium tide (see e.g. Ogilvie 2014; Villaver et al. 2014). The impact of inertial waves in the convective envelope (see e.g. Bolmont \& Mathis 2016) and dynamical tides in the radiative envelope of main-sequence models (Zahn 1975) can be safely neglected in the present computations because the convective envelope of red giants rotate slowly and the separation between the planet and the star (initial semi-major axis of $0.5 \mathrm{au}$ ) is relatively large.

As shown in Sect. 4, a planet engulfment may strongly accelerate the convective envelope of a red giant. When rotation is high enough, it may, through Coriolis acceleration, create differential rotation and helical turbulence in the convective zone, which is required for a dynamo process (the so-called $\alpha$ and $\omega$ effects, respectively; see e.g. Sect. 3.2.2 in Charbonneau 2013). To determine whether Coriolis acceleration is strong enough to create differential rotation, a dimensionless number called Rossby number can be defined as

$\mathrm{Ro} \equiv P_{\mathrm{rot}} / t_{\mathrm{tov}}$,

where $P_{\text {rot }}$ is the stellar spin period and $t_{\text {tov }}$ the convective turnover time. In general, Ro $<1$ indicates the regime where the Coriolis acceleration strongly modifies convective flows and turbulence. We can readily compute the evolution of this number from our rotating stellar models using the expression for the convective turn-over time given by Eq. (4) in Rasio et al. (1996).

An exact theory linking rotation and magnetic field is still lacking, but an empirical relation showing that the surface magnetic field $(B)$ of red giants increases when the Rossby number decreases has recently been found for solar-type and lowmass stars (Vidotto et al. 2014; Aurière et al. 2015). We here use the relation given by Aurière et al. (2015) for red-giant stars: $\lg (B)=-0.85 * \lg ($ Ro $)+0.51$.

Once a sufficiently strong surface magnetic field is generated, it may force the stellar wind to co-rotate with the star. This in turn may lead to a torque on the convective envelope. This is the essence of wind magnetic braking. The loss of angular momentum by this process is first estimated following ud-Doula \& Owocki (2002) and ud-Doula et al. (2008),

$\frac{\mathrm{d} J}{\mathrm{~d} t}=\frac{2}{3} \dot{M} \Omega R^{2}\left[0.29+\left(\eta_{*}+0.25\right)^{1 / 4}\right]^{2}$,

where $J$ is the angular momentum of the convective envelope, $\dot{M}$ the mass-loss rate given by Reimers (1975), $\Omega$, the angular surface velocity, $R$ the stellar radius, and $\eta_{*}$ the magnetic confinement parameter (ud-Doula \& Owocki 2002) defined by $\eta_{*} \equiv B^{2} R^{2} / \dot{M} v_{\infty}$, where $B$ is the surface magnetic field at the equator and $v_{\infty}$ the terminal wind velocity. Winds of red giant stars are expected to be slow, with observed terminal wind velocities of between 30 and $70 \mathrm{~km} \mathrm{~s}^{-1}$ (Robinson et al. 1998). For our models here, we chose $v_{\infty}=50 \mathrm{~km} \mathrm{~s}^{-1}$. Except for $v_{\infty}$, the other quantities were taken from stellar evolution models.

Equation (2) was deduced for hot massive stars from 2D MHD simulations of magnetic wind confinement models where a rotation-aligned dipole magnetic field is considered. In the case of red giants, one notable difference, however, is the ionization fraction of the wind, which is of course much lower for red giants than for the hot stars. The lower the ionization fraction, the lower the coupling between the wind and the magnetic field, probably, and thus the weaker the wind braking. To account for this effect, we computed the loss of angular momentum in our model $(\mathrm{d} L / \mathrm{d} t)$ using a parametric approach by introducing a wind braking efficiency factor $f \leq 1$ so that $\mathrm{d} L / \mathrm{d} t=f \mathrm{~d} J / \mathrm{d} t$, where $\mathrm{d} J / \mathrm{d} t$ is given by Eq. (2).

Because Eq. (2) was deduced for hot massive stars, the question is whether it can be used for red giants with an external convective envelope. In principle, this can be the case if the formalism is not too sensitive to the wind-driving mechanism. To check this point, we also used the formalism of Matt et al. (2012), which has been obtained for solar-type stars. The loss of angular momentum according to Matt et al. (2012) is then given by

$\frac{\mathrm{d} J}{\mathrm{~d} t}=\frac{K_{1}^{2}}{(2 G)^{\mathrm{m}}} B^{4 \mathrm{~m}} \dot{M}^{1-2 \mathrm{~m}} \frac{R^{5 \mathrm{~m}+2}}{M^{\mathrm{m}}} \frac{\Omega}{\left(K_{2}^{2}+0.5 s_{\star}^{2}\right)^{\mathrm{m}}}$,

where $K_{1}=1.3$ and $K_{2}=0.0506, G$ is the gravitational constant, $m=0.2177, M$ is the mass of the star, and $s_{\star}=\Omega R^{3 / 2}(G M)^{-1 / 2}$ (see Matt et al. 2012, for more details). For Eq. (2) an efficiency factor $f$ is used to change the braking efficiency.

\section{Evolution of the Rossby number}

Planet engulfment has a very significant impact on the surface rotation of red giants. We show this in the left panel of Fig. 1, where we plot the evolution of surface rotation velocities as a function of surface gravity for $1.7 M_{\odot}$ stellar models with various initial rotation rates, as indicated in the caption. It then compares what happens when a $15 M_{\mathrm{J}}$ planet is engulfed (at a $\log g$ of around 2.2) by an initially slowly rotating $1.7 M_{\odot}$ star. Clearly, the engulfment leads to a spin-up at a level that cannot be reached by single-star models regardless of their initial rotation rates (Paper II). If a lower mass planet is considered, then this will change the time at which the engulfment occurs, shifting it to lower gravities, which will produce a lower increase in the surface velocity (see Paper II). The same occurs when the mass of the planet is kept constant but the initial distance to the star is increased.

The right panel of Fig. 1 shows the evolution of the Rossby number for the same models. Models without engulfment have a phase where Ro $<1$ at the base of the red giant branch when the star rotates sufficiently fast for Ro to decrease below 1 . More quantitatively, when we initially consider the fastest rotating model, Ro $<1$ only for surface gravities higher than about 2, that is, at the base of the red giant branch. When an engulfment occurs, a quite different evolution of Ro is evident. At the time of engulfment, the Rossby number becomes suddenly smaller than 1. After the engulfment, the star continues to expand and the magnetic braking causes the Rossby number to increase again.

When we compare models with and without engulfment, the most striking difference is that models with planet engulfment produce Ro $<1$ at lower surface gravities (between 1.2 and 2.2), that is, at more advanced stages along the red giant branch star. In this range of surface gravities, no single-star model, regardless of its initial rotation, predicts $\mathrm{Ro}<1$. This suggests that such a model can hardly produce an observable magnetic field. 

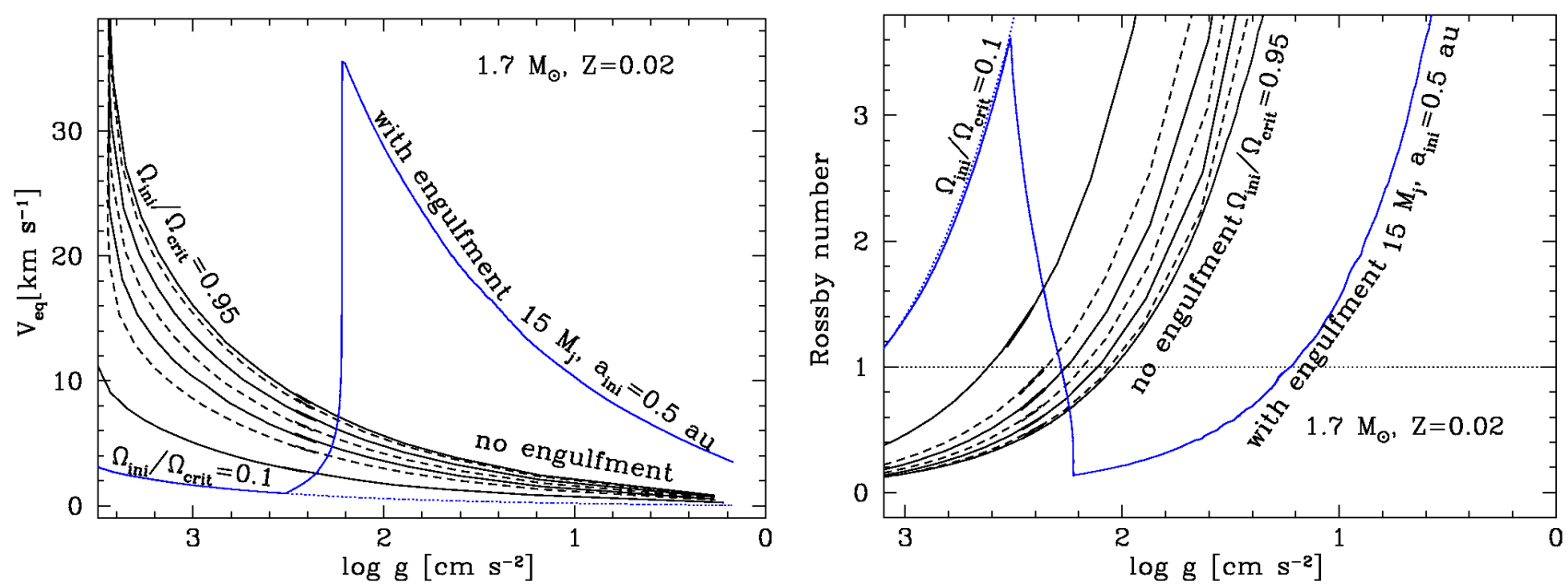

Fig. 1. Left panel: predicted evolution of the surface velocity as a function of the surface gravity for $1.7 M_{\odot}$ stellar models along the red giant branch with and without planet engulfment. Different initial rotations on the ZAMS are considered for models without engulfment: $\Omega_{\text {ini }} / \Omega_{\text {crit }}=0.3$, $0.5,0.6,0.7,0.8,0.9$, and 0.95 (alternate black continuous and dashed lines). The time-averaged surface velocities during the main-sequence phase of the models vary between about 30 and $240 \mathrm{~km} \mathrm{~s}^{-1}$. The model with planet engulfment (blue continuous line, the dotted blue line is the model without engulfment) initially had $\Omega_{\text {ini }} / \Omega_{\text {crit }}=0.1$ and a $15 M_{\mathrm{J}}$ planet orbiting at a distance equal to 0.5 au. The engulfment occurs when the surface gravity of the star is around 2.2. Right panel: evolution of the Rossby number as a function of the surface gravity for the same stellar models along the red giant branch with and without a planet engulfment.
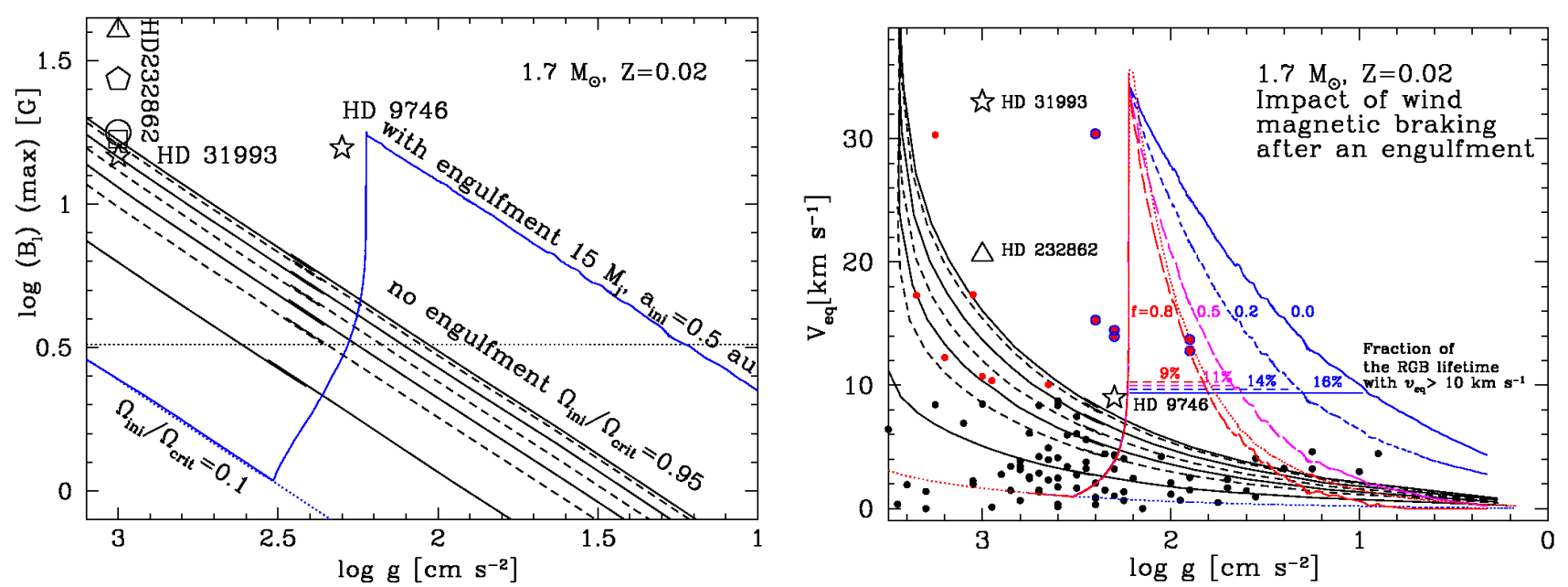

Fig. 2. Left panel: predicted evolution of the surface magnetic field as a function of the surface gravity for $1.7 M_{\odot}$ stellar models along the red giant branch with and without planet engulfment. The models are the same as in Fig. 1. Above the horizontal dashed line, Ro $<1$. The two empty stars are observations from Aurière et al. (2015). The other points correspond to the star HD 232862 observed on four consecutive days by Lèbre et al. (2009; the triangle is the first observation, then the square, pentagon, and the circle). Right panel: evolution of the surface rotation as a function of the surface gravity for the same models as shown in Fig. 1, with in additional cases where magnetic braking laws with various efficiencies have been accounted for after the engulfment. The dashed blue, magenta, and red curves correspond to values of $f$ equal to $0.2,0.5$, and 0.8 using Eq. (2) (see text). The red dotted line is obtained using Eq. (3) with $f=0.5$. The continuous blue curve corresponds to the case $f=0$. We have indicated how the duration of the period during which the surface velocity is above $10 \mathrm{~km} \mathrm{~s}^{-1}$ varies as a function of the strength of the magnetic braking. The same three observations as those indicated in the left panel are shown. The dots show the observations by Carlberg et al. (2012), the black circles show the stars with a $v \sin i<10 \mathrm{~km} \mathrm{~s}^{-1}$, the filled red- and blue-circled magenta points have $v \sin i>10 \mathrm{~km} \mathrm{~s}{ }^{-1}$. The blue-circled magenta points correspond to stars (HD 31994, Tyc 0347-00762-1, Tyc 5904-00513-1, and Tyc 6054-01204-1) whose surface velocity cannot be explained by any reasonable model for single stars (Paper II).

\section{Planet engulfment and surface magnetic field}

We now study the magnetic field generation for our models. The left panel of Fig. 2 shows the predicted evolution of the surface magnetic field as a function of the surface gravity for $1.7 M_{\odot}$ stellar models with and without planet engulfment. Surface magnetic fields of up to a few tens of Gauss could be triggered by an engulfment at surface gravities where such fields are otherwise not expected. Even for the initially faster rotating stars that evolved in isolation, no significant magnetic field is expected for $\log g<2$. Thus, a strong magnetic field at low gravities together with a high surface velocities are strong signs of a past engulfment. Of course, a planet with too low a mass or a planet initially orbiting the star at too large a distance will only lead to a weak magnetic field that will not be observable.

The question here might be asked whether the strong magnetic field linked to the fast rotation might prevent the star from 
keeping trace of the past engulfment high rotation rate for a sufficiently long time to be an observable feature. When our $1.7 M_{\odot}$ reaches its peak surface velocity at the time of engulfment, the surface magnetic field is around $20 \mathrm{G}$. With such a strong field, the shortest wind magnetic braking timescale (obtained using $f=1$ ) is 20 Myr. Since the duration from the engulfment to the tip of the red giant branch is about $34 \mathrm{Myr}$, wind magnetic braking is expected to significantly affect the evolution of the surface velocity (of course, a value of $f=1$ is most likely an overestimate caused by the low ionization fraction of the wind).

In the right panel of Fig. 2 we show the results of various braking laws of the type given by Eq. (2), with different wind braking efficiencies $f$, as indicated in the caption. The wind magnetic braking was applied only to the case with engulfment.

The magnetic braking does not change the maximum velocity reached by the engulfment. The rise is too short for this braking to have any effect. After the engulfment, the stronger the braking, the more rapidly the decrease in surface velocity. Without magnetic braking, the star retains a surface velocity above $10 \mathrm{~km} \mathrm{~s}^{-1}$ for 29 Myr. When $f$ equals $0.2,0.5$, or 0.8 , this duration reduces to 25,20 , and $16 \mathrm{Myr}$, respectively. Compared to the whole red giant branch lifetime, which is about $181 \mathrm{Myr}$, these values correspond to fractions equal to $16 \%(f=0), 14 \%$ $(f=0.2), 11 \%(f=0.5)$, and $9 \%(f=0.8)$, thus still quite substantial values. This means that even though the wind magnetic braking counteracts the effect of the engulfment on the surface velocities, planet engulfment remains a strong candidate for explaining the observed fast-rotating red giants.

All these results were obtained by using the magnetic braking formalism of ud-Doula \& Owocki (2002) and ud-Doula et al. (2008; Eq. (2)). A model computed with the formalism of Matt et al. (2012; Eq. (3)) is shown by the red dotted line in the right panel of Fig. 2. A value of $f$ equal to 0.5 was used in this case. The two braking formalisms lead to very similar results (only a slight increase of the global efficiency of the braking is seen when Eq. (3) is used). This can be understood by comparing for instance the expressions for the Alfvén radius in the two cases (Eq. (19) in ud-Doula et al. 2008 and Eq. (6) in Matt et al. 2012): for red giants, the term related to the magnetic confinement parameter dominates and the dependence of the Alfvén radius on this term is nearly identical in both formalisms (a value of $m$ of 0.2177 for Matt et al. 2012, and 0.25 for ud-Doula et al. 2008).

Wind magnetic braking would also affect the evolution of the surface velocities of our models with no engulfment. These stars, as shown above, may also develop a surface magnetic field at the base of the red giant branch (see right panel of Fig. 1 and the left panel of Fig. 2), which, at its turn, may drive some wind magnetic braking. This would shift the curves corresponding to no engulfment in the surface velocity downward compared to the surface gravity plots (see the left panel of Fig. 1 and the right panel of Fig. 2), but would not much affect what occurs during the engulfment. For the case considered here, the quantity of angular momentum added by the planet is so large that the initial surface rotation of the star has little influence.

Wind magnetic braking during the red giant phase would change the interpretation of the observed surface rotations of red giants stars. Wind magnetic braking would shift to lower values the maximum surface velocity that can be reached at a given surface gravity by an initial mass model that evolves without interaction (tides or engulfment). The maximum surface velocity is obtained by considering the highest possible initial rotation on the ZAMS and assuming solid-body rotation. Any stars presenting higher surface rotations than this limit are very strong candidates for having experienced planet engulfment (see more in Paper II). Since wind magnetic braking would lower this limit, it would allow more observed stars to lie above it and thus, as indicated above, would enlarge the size of stars whose surface rotation needs an interaction to be explained. A too efficient magnetic braking would probably increase the number of candidates that would have had to experience such interactions by too much. This point will be discussed in a more extended future work.

Although it is beyond the scope of the present work to make detailed comparisons with observations, we discuss below three stars that are interesting candidates for having engulfed a planet, and that have masses around $1.7 \pm 0.3 M_{\odot}$, that is, they are similar to the models discussed here. The positions of these stars in the magnetic field versus $\log g$ and in the surface velocity versus $\log g$ plots are indicated in Fig. 2. The daily variation of the magnetic field of HD 232862 may be due to the change of the viewing angle when the star rotates. These three stars have surface magnetic fields and rotations that are high enough for being good candidates for a planet engulfment. Two of them (HD 31993 and 232862) might also be explained by assuming initially very fast-rotating stars, but the probability of such extreme initial conditions is quite low so that an interaction remains a more reasonable explanation for the currently observed properties. For these two stars, the engulfment of a $15 M_{\mathrm{J}}$ planet that began orbiting the star at 0.5 au occurs at a gravity that is too low to explain their properties. This would indicate a higher mass planet or a shorter initial distance between planet and star. The magnetic field of HD 9746 may well be explained by the case of engulfment shown in the figures. Its $v \sin i$ is equal to $9 \mathrm{~km} \mathrm{~s}^{-1}$ (Balachandran et al. 2000). Our models would predict a value $v$ around $30 \mathrm{~km} \mathrm{~s}^{-1}$.

Planet-induced magnetic field theory might be tested by checking whether those stars whose high surface velocities cannot be explained by stellar models evolving in isolation truly present measurable magnetic fields. Typically, stars such as HD 31994, Tyc 0347-00762-1, Tyc 5904-00513-1, and Tyc 6054-01204-1 (the blue-circled magenta points in Fig. 2) are interesting candidates.

\section{Conclusion}

A planet engulfment can increase stellar rotation to such an extent that an observable surface magnetic field can develop. Planet engulfment might explain the strong magnetic field in the upper part of the red giant branch, where stars without interaction rotate too slowly to allow a dynamo to create such strong fields. Some good candidate stars show both high surface rotation rates and high surface magnetic fields that are compatible with an engulfment. Likewise, some fast-rotating red giants may be found to show some surface magnetic fields if our present models are correct (for instance HD 31994, Tyc 0347-00762-1, Tyc 590400513-1, and Tyc 6054-01204-1).

The planet-induced magnetic fields are strong enough to slow the star down by wind magnetic braking. This will reduce the time that the surface velocity of a star can be maintained above a given limit. However, for moderate couplings, the wind magnetic braking effect does not disrupt the production of observable (i.e. sufficiently long-lasting) fast-rotating red giants by planet engulfment. This seems compatible with recent results that suggested a low efficiency of surface magnetic braking for stars more evolved than the Sun (van Saders et al. 2016).

The increased stellar rotation and magnetic field generation linked to planet engulfment can in principle operate on the wind 
and might increase the mass loss. Whether this mechanism can explain the high mass-loss rates needed to form hot subdwarf stars that are singles is an interesting question that needs to be explored in the future.

Acknowledgements. We would like to thank Stéphane Mathis for his valuable comments and suggestions. The project has been supported by Swiss National Science Foundation grants 200021-138016, 200020-160119 and 200020-15710. E.V. acknowledges support from the Spanish Ministerio de Economía y Competitividad under grant AYA2014-55840P. A.uD. acknowledges support by NASA through Chandra Award numbers GO5-16005X, AR617002C and G06-17007B issued by the Chandra X-ray Observatory Center which is operated by the Smithsonian Astrophysical Observatory for and behalf of NASA under contract NAS8- 03060.

\section{References}

Adamów, M., Niedzielski, A., Villaver, E., Nowak, G., \& Wolszczan, A. 2012, ApJ, 754, L15

Alexander, J. B. 1967, The Observatory, 87, 238

Aurière, M., Konstantinova-Antova, R., Charbonnel, C., et al. 2015, A\&A, 574, A90

Balachandran, S. C., Fekel, F. C., Henry, G. W., \& Uitenbroek, H. 2000, ApJ, 542,978

Bolmont, E., \& Mathis, S. 2016, Celestial Mechanics and Dynamical Astronomy, [arXiv: 1603.06268]

Carlberg, J. K. 2014, AJ, 147, 138

Carlberg, J. K., Majewski, S. R., \& Arras, P. 2009, ApJ, 700, 832

Carlberg, J. K., Smith, V. V., Cunha, K., Majewski, S. R., \& Rood, R. T. 2010, ApJ, 723, L103

Carlberg, J. K., Cunha, K., Smith, V. V., \& Majewski, S. R. 2012, ApJ, 757, 109
Charbonneau, P. 2013, Solar and Stellar Dynamos: Saas-Fee Advanced Course 39 Swiss Society for Astrophysics and Astronomy, Saas-Fee Advanced Courses, (Berlin Heidelberg: Springer-Verlag), 39

Fekel, F. C., \& Balachandran, S. 1993, ApJ, 403, 708

Kunitomo, M., Ikoma, M., Sato, B., Katsuta, Y., \& Ida, S. 2011, ApJ, 737, 66

Lèbre, A., Palacios, A., Do Nascimento, Jr., J. D., et al. 2009, A\&A, 504, 1011

Matt, S. P., MacGregor, K. B., Pinsonneault, M. H., \& Greene, T. P. 2012, ApJ, 754, L26

Nordhaus, J., Spiegel, D. S., Ibgui, L., Goodman, J., \& Burrows, A. 2010, MNRAS, 408, 631

Ogilvie, G. I. 2014, ARA\&A, 52, 171

Privitera, G., Meynet, G., Eggenberger, P., et al. 2016a, A\&A, 591, A45

Privitera, G., Meynet, G., Eggenberger, P., et al. 2016b, A\&A, in press, DOI: $10.1051 / 0004-6361 / 201628758$

Rasio, F. A., Tout, C. A., Lubow, S. H., \& Livio, M. 1996, ApJ, 470, 1187

Reimers, D. 1975, Mem. Soc. Roy. Sci. Liège, 8, 369

Robinson, R. D., Carpenter, K. G., \& Brown, A. 1998, ApJ, 503, 396

Sandquist, E., Taam, R. E., Lin, D. N. C., \& Burkert, A. 1998, ApJ, 506, L65

Sandquist, E. L., Dokter, J. J., Lin, D. N. C., \& Mardling, R. A. 2002, ApJ, 572, 1012

Siess, L., \& Livio, M. 1999a, MNRAS, 304, 925

Siess, L., \& Livio, M. 1999b, MNRAS, 308, 1133

Soker, N., Livio, M., \& Harpaz, A. 1984, MNRAS, 210, 189

ud-Doula, A., \& Owocki, S. P. 2002, ApJ, 576, 413

ud-Doula, A., Owocki, S. P., \& Townsend, R. H. D. 2008, MNRAS, 385, 97 van Saders, J. L., Ceillier, T., Metcalfe, T. S., et al. 2016, Nature, 529, 181

Vidotto, A. A., Gregory, S. G., Jardine, M., et al. 2014, MNRAS, 441, 2361

Villaver, E., \& Livio, M. 2007, ApJ, 661, 1192

Villaver, E., \& Livio, M. 2009, ApJ, 705, L81

Villaver, E., Livio, M., Mustill, A. J., \& Siess, L. 2014, ApJ, 794, 3

Zahn, J.-P. 1975, A\&A, 41, 329

Zahn, J.-P. 1977, A\&A, 57, 383

Zahn, J.-P. 1989, A\&A, 220, 112

Zahn, J.-P. 1992, A\&A, 265, 115 